\title{
openheart Vessel fractional flow reserve in assessment of non-culprit lesions in ST elevation myocardial infarction
}

\author{
Chun Chin Chang (D) , , 1,2,3 Ming Ju Chuang, ${ }^{1,3}$ Yin Hao Lee, ${ }^{1,3}$ Yi Lin Tsai, ${ }^{1,3}$ \\ Ya Wen Lu, ${ }^{1,3}$ Ruey Hsing Chou, ${ }^{1,2,3,5}$ Cheng Hsueh Wu, ${ }^{1,3,5}$ Tse Min Lu, ${ }^{1,2,6}$ \\ Po Hsun Huang, ${ }^{1,2,3,5}$ Shing Jong Lin ${ }^{1,2,3,7}$
}

To cite: Chang CC, Chuang MJ, Lee $\mathrm{YH}$, et al. Vessel fractional flow reserve in assessment of non-culprit lesions in ST elevation myocardial infarction. Open Heart 2021;8:e001691. doi:10.1136/ openhrt-2021-00169

Received 14 April 2021 Accepted 13 July 2021

D Check for updates

(C) Author(s) (or their employer(s)) 2021. Re-use permitted under CC BY-NC. No commercial re-use. See rights and permissions. Published by BMJ.

${ }^{1}$ Department of Internal

Medicine, Division of Cardiology, Taipei Veterans General Hospital Taipei City, Taiwan

${ }^{2}$ Institute of Clinical Medicine, National Yang Ming Chiao Tung University, Taipei, Taiwan

${ }^{3}$ Cardiovascular Research Center, National Yang Ming Chiao Tung University, Taipei, Taiwan

${ }^{4}$ Department of Cardiology, Thoraxcenter, Erasmus Medical Center, Rotterdam, Netherlands ${ }^{5}$ Department of Critical Care Medicine, Taipei Veterans General Hospital, Taipei, Taiwan ${ }^{6} \mathrm{Healthcare}$ and services center, Taipei Veterans General Hospital, Taipei, Taiwan

${ }^{7}$ Taipei Heart Institute, Taipei Medical University, Taipei,

Taiwan

Correspondence to Professor Po Hsun Huang; huangbsvgh@gmail.com

\section{ABSTRACT}

Objectives We sought to evaluate the physiology of non-culprit lesions by using vessel fractional flow reserve (vFFR) among patients with ST elevation myocardial infarction (STEMI) and multivessel disease (MVD). Methods From January 2017 to December 2019, 354 patients with STEMI in the Taipei Veterans General Hospital Acute Myocardial Infarction Registry were screened. Patients who underwent successful primary percutaneous coronary intervention (PCI) for culprit lesions, with at least one non-culprit lesion with stenosis of $\geq 50 \%$, were eligible. vFFR was computed retrospectively.

Results A total of 156 patients with 217 non-culprit lesions were eligible for this study. Aortic root pressure and two good angiograms were available for 139 non-culprit lesions for vFFR analysis. Based on the vFFR analysis, 59 non-culprit lesions (43.2\%) had a vFFR value $>0.80$, and $\mathrm{PCl}$ was deferred in 45 lesions (76.3\%). Meanwhile, 80 non-culprit lesions $(56.8 \%)$ had a vFFR value $\leq 0.80$; however, $\mathrm{PCl}$ was only performed in 31 lesions $(38.7 \%)$ $(p=0.142)$. The incidence of vessel-oriented composite endpoint was numerically higher in non-culprit lesions with vFFR $\leq 0.80$ than those with vFFR $>0.80(6.3 \%$ vs $1.7 \%$, HR: $3.59,95 \%$ Cl: 0.42 to $30.8, p=0.243$ ). Conclusion Functional incomplete revascularisation is common among patients with STEMI and MVD. The adoption of vFFR to assess non-culprit lesions may reclassify the coronary revascularisation strategy that is usually guided by angiography only in this acute setting.

\section{INTRODUCTION}

Coronary angiography and primary percutaneous coronary intervention (PCI) are the current standards of care for managing ST elevation myocardial infarction (STEMI). The COMPLETE trial indicated that in patients with STEMI and multivessel disease (MVD), complete revascularisation for nonculprit lesions with stenosis $>70 \%$ was superior to culprit-lesion-only PCI. ${ }^{1}$ Recently, DANAMI-3-PRIMULTI and COMPAREACUTE, two large randomised clinical studies, have demonstrated that fractional flow reserve (FFR)-guided PCI of non-culprit

\section{Key questions}

What is already known about this subject?

- Fractional flow reserve (FFR)-guided percutaneous coronary intervention (PCl) of non-culprit lesions in patients with ST elevation myocardial infarction (STEMI) had significantly lower adverse event rates compared to culprit-lesion-only PCl. Nevertheless, FFR is rarely used in clinical practice during primary $\mathrm{PCl}$ for patients with STEMI and multivessel disease (MVD).

What does this study add?

- We demonstrated that functional incomplete revascularisation is common in angiography-guided $\mathrm{PCl}$ in patients with STEMI and MVD. The vessel FFR (vFFR) can be used to assess non-culprit lesions based on angiograms of the index procedure in a post hoc manner. vFFR of non-culprit lesions was highly correlated in the acute and staged procedures.

How might this impact on clinical practice?

- vFFR may increase the adoption rate of physiology assessment for non-culprit lesions in patients with STEMI and MVD. The use of vFFR may reduce the number of unnecessary downstream invasive coronary angiography procedures and may lead to an increase in functional complete revascularisation.

lesions in patients with STEMI had significantly lower adverse event rates compared with culprit-lesion-only PCI. $^{2}{ }^{3}$ Nevertheless, FFR is rarely used in clinical practice in primary PCI for patients with STEMI and MVD. The use of FFR is limited by the necessity of intravenous or intracoronary adenosine infusion and additional equipment. Therefore, the decision of revascularisation for non-culprit lesions in patients with STEMI is typically based on coronary angiograms of the index procedure, or a staged procedure is needed at the operator's discretion. Based on the literature, further study is needed to assess the implications of coronary physiology in this setting. 
Currently, coronary physiology can be assessed without medications or a pressure wire. Vessel FFR (vFFR) is a novel physiological index that is calculated based on computational fluid dynamics and a three-dimensional reconstruction model. The Fast Assessment of STenosis severity (FAST) Study showed a strong linear correlation between FFR and vFFR, with good reproducibility and high diagnostic accuracy. ${ }^{4}$ This angiography-derived functional assessment can be performed ad hoc or retrospectively when treating patients with STEMI and MVD. In this context, we sought to evaluate vFFR values of nonculprit lesions and investigate the concordance rate of revascularisation strategy that is suggested by vFFR and the actual treatment performed in patients with STEMI and MVD.

\section{METHODS}

\section{Study design and participants}

The present study was an observational, retrospective, single-centre study. From January 2017 to December 2019, patients presenting with STEMI at Taipei Veterans General Hospital were screened. STEMI was defined according to the Third Universal Myocardial Infarction definition. ${ }^{5}$ Patients who underwent successful primary PCI for culprit lesions, with at least one non-culprit lesion with stenosis of $\geq 50 \%$ with a reference vessel diameter $\geq 2.0 \mathrm{~mm}$ (by visual estimation) on coronary angiography, were eligible. Patients who presented with cardiac arrest, profound cardiogenic shock or ostial or left main non-culprit lesions were excluded. The decision for treatment of non-culprit lesions was based on coronary angiograms and clinical factors. PCIs for nonculprit lesions were performed at the time of primary PCI or in a staged procedure. A staged procedure was defined as a planned intervention, and the procedure should be performed electively within 16 weeks after the primary PCI. ${ }^{6}$ The study was approved by the Taipei Veterans General Hospital's research ethics committee and was conducted in accordance with the Declaration of Helsinki.

\section{vFFR analysis}

The vFFR analysis was performed by independent analysts using CAAS Workstation (V.8.2; Pie Medical Imaging, Maastricht, the Netherlands) and was based on previous reports of image acquisition and processing. ${ }^{4}$ Briefly, two angiograms with at least a $30^{\circ}$ difference in rotation/angulation are required to create a 3D reconstruction of the coronary artery. Coronary angiography was performed using a conventional radiation mode (15 frames per second and 15 pulses per second). The software contour detection was performed semiautomatically, delineating the vessel contour from the ostium to a distal position, with manual correction performed when needed. A 3D quantitative coronary angiography (QCA) and vFFR values were automatically generated.

\section{Clinical endpoints per vessel}

The primary endpoint of this study was the vessel-oriented composite endpoint (VOCE) during clinical follow-up, defined as a composite of vessel-related cardiac death, vessel-related myocardial infarction (MI) and target vessel revascularisation (TVR). Cardiac death was defined as death resulting from cardiovascular causes (eg, acute MI, cardiac death or unwitnessed death or heart failure) ${ }^{7}$ MI was defined according to the Third Universal Myocardial Infarction definition. ${ }^{5}$ TVR was defined as any repeat percutaneous intervention or surgical bypass of any segment of the target vessel including the target lesion. ${ }^{7}$ VOCE was analysed in a hierarchical manner.

Clinical events were counted per vessel and were classified as vessel related or not vessel related. If the patient was classified as cardiac death without a clear correlation to a specific vessel (eg, heart failure or unwitnessed death) at follow-up, the worst scenario was considered, and the death was adjudicated as a vessel-related cardiac death. Whenever an MI or revascularisation occurred, angiograms at event and medical records were reviewed by investigators for clinical event adjudication.

\section{Statistical methods}

Categorical variables are presented as percentages and numbers. Continuous variables are presented as the mean \pm SD. Pearson's correlation coefficient and Cohen's kappa coefficient were used to evaluate the correlation and agreement of vFFR values at two different times (primary PCI vs staged PCI). Survival curves were constructed using Kaplan-Meier estimates and the logrank test to compare between-group differences. An HR was reported with 95\% CIs based on the Cox regression model. A two-sided $p$ value of $<0.05$ was considered statistically significant. Data were analysed using SPSS software (V.25).

\section{RESULTS}

A total of 354 patients who presented with STEMI were screened, and 156 patients with 217 non-culprit lesions were eligible for this study. Among these patients, aortic root pressure and two good angiograms were available for vFFR analysis of 139 non-culprit lesions. The retrospective analysability was $64 \%$, and the reasons of being nonanalysable were mainly due to no appropriate two projections to perform vFFR analysis for non-culprit lesions. A representative case of $\mathrm{vFFR}$ analysis is shown in figure 1. The baseline characteristics of the eligible patients are shown in table 1 . The mean age was $65.8 \pm 14.5$ years, $86.5 \%$ were men and $34.6 \%$ had diabetes. Most patients $(84.6 \%)$ only underwent PCI for culprit lesions in the index procedure, whereas $14.7 \%$ also received PCI for non-culprit lesions during the index procedure. Drugeluting stents were deployed in $76.3 \%$ of patients.

The locations of non-culprit lesions and 3D QCA data are provided in table 2. The mean minimal lumen diameter, percent diameter stenosis and 


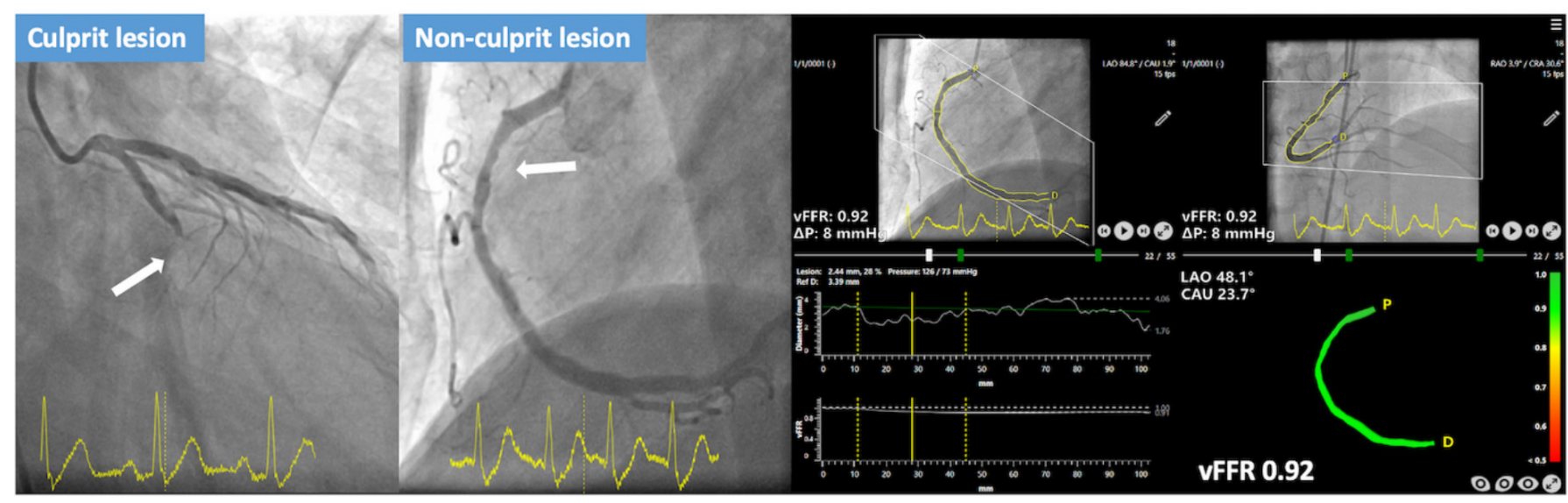

Figure 1 A representative case of vFFR analysis. vFFR=vessel fraction flow reserve.

Table 1 Baseline characteristics of patients

\begin{tabular}{lc}
\hline Baseline characteristics & $\begin{array}{c}\text { All patients } \\
\text { (n=156) }\end{array}$ \\
\hline Age (years) & $65.8 \pm 14.5$ \\
\hline Sex & \\
\hline Man & $135(86.5 \%)$ \\
\hline Woman & $21(13.5 \%)$ \\
\hline Medical history & \\
\hline Hypertension & $79(50.6 \%)$ \\
\hline Diabetes mellitus & $54(34.6 \%)$ \\
\hline Chronic kidney disease & $24(15.4 \%)$ \\
\hline Previous myocardial infarction & $7(4.5 \%)$ \\
\hline Previous percutaneous coronary intervention (PCI) & $13(8.3 \%)$ \\
\hline Previous coronary bypass grafting & $0(0.0 \%)$ \\
\hline Heart failure & $2(1.3 \%)$ \\
\hline Location of culprit lesion & \\
\hline Left anterior descending artery & $78(50 \%)$ \\
\hline Left circumflex & $14(9 \%)$ \\
\hline Right coronary artery & $64(41 \%)$ \\
\hline Number of vessel treated in primary PCI & $132(84.6 \%)$ \\
\hline 1 (culprit lesion only) & $23(14.7 \%)$ \\
\hline 2 & $1(0.7 \%)$ \\
\hline Type of stent used in primary PCl & \\
\hline DES & $29(18.6 \%)$ \\
\hline BMS & \\
\hline DES and BMS & $(1.3 \%)$ \\
\hline
\end{tabular}

Data were mean \pm SD or number (\%).

BMS, bare-metal stent; BRS, bioresorbable scaffold; DES, drugeluting stent. obstruction length were $1.44 \pm 0.51 \mathrm{~mm}, 44.5 \% \pm 12.5 \%$, and $19.2 \pm 12.5 \mathrm{~mm}$, respectively. The mean vFFR values of non-culprit lesions included the following: left anterior descending artery, $0.72 \pm 0.13$; left circumflex artery, $0.74 \pm 0.17$; and right coronary artery, $0.81 \pm 0.19$, respectively (table 3 ). Based on the vFFR analysis, 59 non-culprit lesions $(43.2 \%)$ had a vFFR value $>0.80$, and PCI was deferred in 45 lesions $(76.3 \%)$. Meanwhile, 80 non-culprit lesions (56.8\%) had a vFFR value $\leq 0.80$; however, PCI was only performed in 31 lesions (38.7\%) ( $\mathrm{p}=0.142)$ (table 4). The concordance rate of revascularisation strategy recommended by the post hoc vFFR was only $54.4 \%$ $(76 / 139)$ with the actual treatment, which was mainly guided by angiography. During a mean follow-up of $672 \pm 412$ days, the incidence of VOCE was numerically higher in non-culprit lesions with a vFFR value $\leq 0.80$ than those $>0.80$ (6.3\% vs $1.7 \%$, HR: $3.59,95 \%$ CI: 0.42 to $30.8, \mathrm{p}=0.243$ ) (figure $\mathbf{2 A}$ ). We further stratified non-culprit lesions into four groups based on PCI deferred or performed. In lesions with a vFFR value

Table 2 Baseline characteristics of non-culprit lesions studied

Non-culprit lesions $(n=139)$

\begin{tabular}{ll}
\hline Location of non-culprit lesions & \\
Left anterior descending artery & $48(34.5 \%)$ \\
Left circumflex & $40(28.8 . \%)$ \\
Right coronary artery & $51(36.7 \%)$ \\
3D quantitative coronary angiography & \\
Diameter stenosis (\%) & $44.5 \pm 12.5$ \\
Reference vessel diameter (mm) & $2.62 \pm 0.70$ \\
Minimal lumen diameter (mm) & $1.44 \pm 0.51$ \\
Obstruction length (mm) & $19.2 \pm 12.5$ \\
\hline
\end{tabular}

Data were number (\%) or mean \pm SD. 
Table 3 Vessel fraction flow reserve (vFFR) values of non-culprit lesions

\begin{tabular}{lllll}
\hline & LAD $(\mathbf{n}=\mathbf{4 8})$ & LCX $(\mathbf{n}=\mathbf{4 1})$ & RCA $(\mathbf{n = 5 0 )}$ & Total $(\mathbf{n}=\mathbf{1 3 9 )}$ \\
\hline vFFR value $>0.8$ & $11(22.9 \%)$ & $15(36.6 \%)$ & $33(66.0 \%)$ & $59(42.4 \%)$ \\
vFFR value $\leq 0.8$ & $37(77.1 \%)$ & $26(63.4 \%)$ & $17(34.0 \%)$ & $80(57.6 \%)$ \\
vFFR value & $0.72 \pm 0.13$ & $0.74 \pm 0.17$ & $0.81 \pm 0.19$ & $0.76 \pm 0.17$ \\
\hline
\end{tabular}

Data were number (\%) or mean \pm SD.

LAD, left anterior descending artery; LCX, left circumflex artery; RCA, right coronary artery; vFFR, vessel fractional flow reserve.

\section{Table 4 Actual management of non-culprit lesions}

\begin{tabular}{lll}
\hline & vFFR value $>0.8$ (n=59 lesions) & vFFR value $\leq 0.8$ (n=80 lesions) \\
\hline PCI was deferred & $45(76.3 \%)$ & $49(61.3 \%)$ \\
PCI was performed in primary PCI & $5(8.5 \%)$ & $8(10.0 \%)$ \\
PCI was performed in staged PCI & $9(15.2 \%)$ & $23(28.7 \%)$ \\
\hline
\end{tabular}

Data was number (\%)

$\mathrm{PCl}$, percutaneous coronary intervention; vFFR, vessel fractional flow reserve.
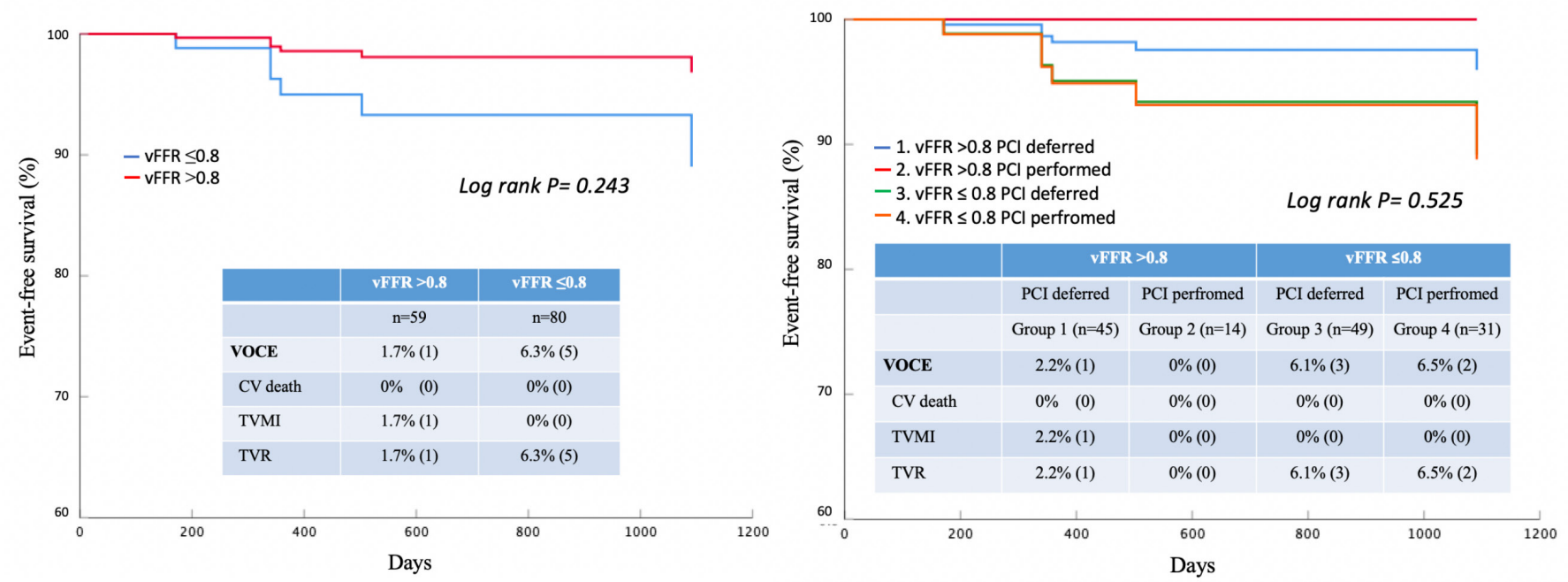

Figure 2 Event-free survival curves. CV=cardiovascular, vFFR=vessel fraction flow reserve, VOCE=vessel-oriented composite endpoint, TVMI=target vessel myocardial infarction and TVR=target vessel revascularisation.

$\leq 0.80$, the incidence of VOCE was similar between the deferred and performed groups (figure 2B).

Among the 32 non-culprit lesions that underwent a staged PCI, vFFR analysis was feasible in 22 lesions. The mean duration between index and staged PCI was $46 \pm 36$ days. The correlation of vFFR of the same non-culprit lesions between primary and staged PCI was significant $(\mathrm{r}=0.822, \mathrm{p}<0.001)$. Similarly, agreement in functional significance was also significant (Cohen's kappa=0.861). The Bland-Altman plots that displayed a mean difference between index and staged vFFR were 0.03 (95\% CI: -0.15 to 0.21 ) (figure 3).

\section{DISCUSSION}

In the present study, we investigated the coronary physiology of non-culprit lesions using vFFR analysis in patients with STEMI and MVD. The main findings of the present study are as follows. In the setting of angiography-guided
PCI, more than half of non-culprit lesions with vFFR $\leq 0.8$ remained with functional incomplete revascularisation. Also, the vFFR of non-culprit lesions measured acutely was highly correlated with vFFR measured subsequently after STEMI. Given that vFFR analysis can be performed acutely or retrospectively, vFFR can be used to assess the coronary physiology of non-culprit lesions after primary PCI.

Previous studies have shown that patients with functional incomplete revascularisation had a significantly higher rate of major adverse cardiac events than those with functional complete revascularisation. ${ }^{8}$ However, the utilisation rate of FFR for assessing intermediate coronary lesions is only about $15 \% .{ }^{910}$ Although randomised trials have demonstrated the benefit of FFR-guided complete revascularisation in patients with STEMI and MVD, it is not realistic to perform FFR measurement for non-culprit lesions acutely in primary PCI. In addition, 

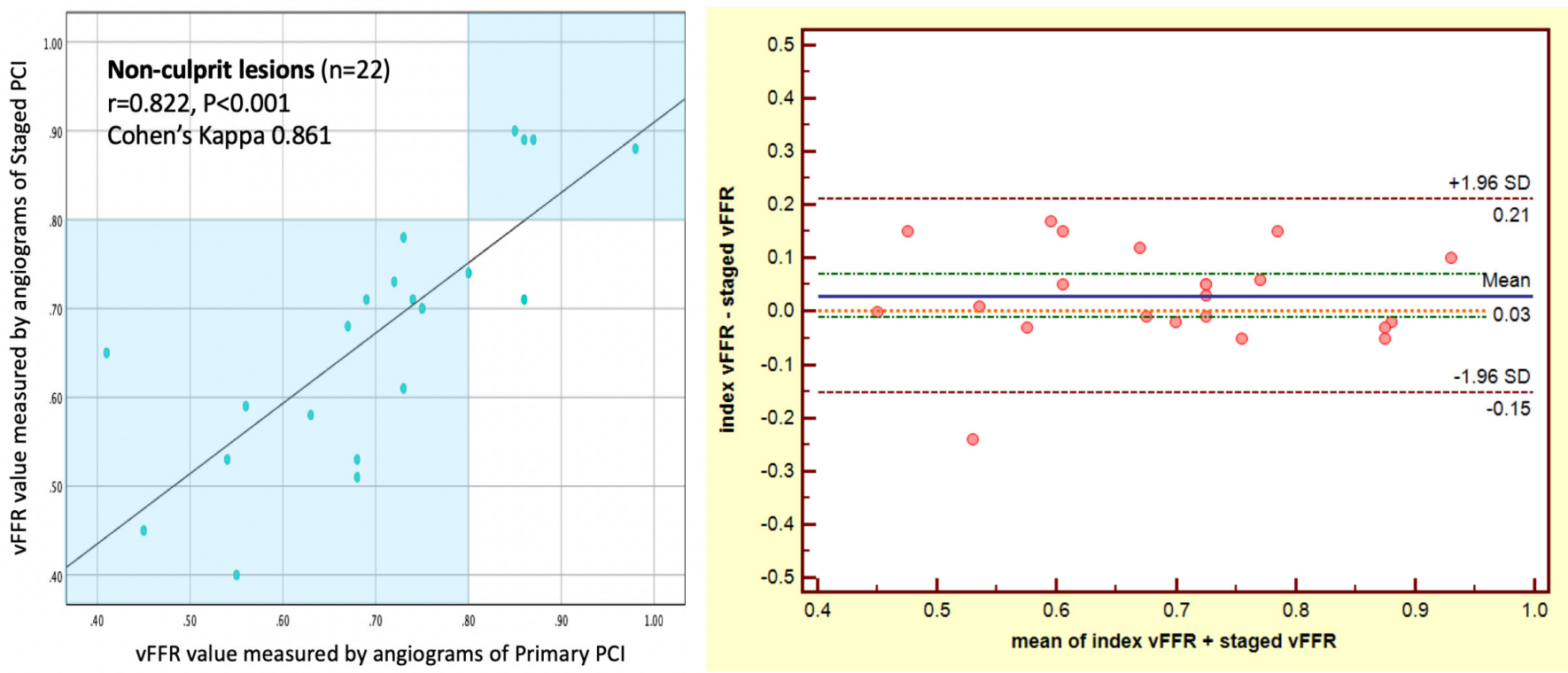

Figure 3 Correlation between vFFR in primary and staged PCI. PCl=percutaneous coronary intervention and vFFR=vessel fraction flow reserve.

the accuracy of wire-based physiological indices may be slightly affected in the setting of acute MI due to disturbed microvascular function. ${ }^{11} 12$ In this setting, angiographyguided decision-making for the treatment of non-culprit lesions was commonly adopted rather than physiologyguided PCI. In the COMPLETE trial, non-culprit lesions with more than $70 \%$ stenosis by visual assessment were treated with PCI directly, while those with a stenosis between $50 \%$ and $70 \%$ were assessed with pressure wire. Physiology-guided revascularisation was used in only $1.4 \%$ of the lesions. ${ }^{1}$ Alternatively, the mismatch between anatomical and functional assessment of coronary lesions has been extensively reported and showed a significant reclassification in indication for revascularisation. ${ }^{13} 14$ In our study, a quarter of non-culprit lesions with vFFR values $>0.8$ underwent $\mathrm{PCI}$, whereas more than half of the lesions with vFFR $\leq 0.8$ were left untreated. Although there are a multitude of reasons for the undertreatment of functionally significant lesions in our cohort, there is a need to implement functional assessment in the management of non-culprit lesions in a more practical way rather than using FFR in primary PCI. In our study, vFFR of non-culprit lesions was highly correlated in the acute and staged procedures. vFFR may be used to assess nonculprit lesions based on angiograms of the index procedure in an ad hoc or post hoc manner. The same type of analysis using quantitative flow ratio (QFR) for nonculprit lesion of STEMI has been reported. ${ }^{15}$ Acute QFR of non-culprit lesions had a good diagnostic performance with both staged QFR and staged FFR. Therefore, the use of angiography-derived FFR, such as vFFR and QFR, may reduce the number of unnecessary downstream invasive coronary angiography procedures and may also lead to an increase in functional complete revascularisation in patients with STEMI and MVD.

QFR and vFFR are both a type of angiographyderived FFR. They are computed using different fluid dynamics models. ${ }^{16}$ A Bayesian meta-analysis has shown that the diagnostic performance of angiography-derived FFR is good with high sensitivity and specificity with pressure wire-based FFR as reference. ${ }^{17}$ There are no differences in accuracy for detecting functionally significant lesions between vFFR, QFR and FFR ${ }_{\text {angio }}$. At variance with FFR or instantaneous wave-free ratio (iFR), the prognostic value of angiography-derived FFR is under investigation. ${ }^{18-20}$ In our study, lesions with vFFR $\leq 0.8$ had a numerically higher rate of VOCE than lesions with vFFR $>0.8$. However, the event rate was relatively low in our study to demonstrate meaningful differences, and potential confounding variables may affect our findings. A large-scale, prospective study with adequate power is demanded to evaluate whether the implementation of vFFR for assessing non-culprit lesions in patients with STEMI and MVD has an influence on clinical outcomes compared with angiography-guided revascularisation.

\section{Limitations}

Our study has several limitations. First, this was a retrospective study; therefore, coronary angiograms were obtained based on routine clinical practice. As such, the retrospective analysability of vFFR for non-culprit lesions was limited in our study since interventionists usually focused on the culprit lesions during the primary PCI. Similarly, the reported analysability of QFR was also low in the same setting. ${ }^{15}$ Further studies with a dedicated 
imaging acquisition protocol are warranted to evaluate the analysability in this acute setting. Also, our results obtained from a single centre with a limited sample size need to be confirmed in future studies. Additionally, FFR was not performed for non-culprit lesions in our study. Therefore, the correlation between FFR and vFFR in patients with acute coronary syndrome warrants further investigation.

\section{CONCLUSION}

Functional incomplete revascularisation is common in real-world practice in patients with STEMI and MVD. The vFFR might be an alternative physiological index used to assess non-culprit lesions in this acute setting, and the adoption of vFFR may reclassify the coronary revascularisation strategy that is guided by angiography.

Acknowledgements All authors would like to thank Jan-Jurre Mordang and Jorn Roijen from Pie Medical Imaging for their technical support.

Contributors CCC, PHH and SJL contributed to the conception and design of the study. CCC, YHL, MJC, YWL, YLT, RHC, CHW and TML contributed to data collection. CCC, YHL and MJC analysed and interpreted the data. CCC, YHL and MJC drafted the report, which was critically revised for important intellectual content by TML, PHH and SJL. All authors approved the final version of the report.

Funding This study was supported in part by research grants from the Novel Bioengineering and Technological Approaches to Solve Two Major Health Problems in Taiwan programme sponsored by the Taiwan Ministry of Science and Technology Academic Excellence Programme (108-2633-B-009-001) and Taipei Veterans General Hospital (VGH-V100E2-002 and VGHUST103-G7-2-1).

Competing interests None declared.

Patient consent for publication Not required.

Ethics approval The study was approved by the Taipei Veterans General Hospital's research ethics committee and was conducted in accordance with the Declaration of Helsinki.

Provenance and peer review Not commissioned; externally peer reviewed.

Data availability statement No data are available.

Open access This is an open access article distributed in accordance with the Creative Commons Attribution Non Commercial (CC BY-NC 4.0) license, which permits others to distribute, remix, adapt, build upon this work non-commercially, and license their derivative works on different terms, provided the original work is properly cited, appropriate credit is given, any changes made indicated, and the use is non-commercial. See: http://creativecommons.org/licenses/by-nc/4.0/.

ORCID iD

Chun Chin Chang http://orcid.org/0000-0003-2799-1185

\section{REFERENCES}

1 Mehta SR, Wood DA, Storey RF, et al. Complete revascularization with multivessel PCl for myocardial infarction. $N$ Engl J Med 2019;381:1411-21.

2 Smits PC, Abdel-Wahab M, Neumann F-J, et al. Fractional flow reserve-guided multivessel angioplasty in myocardial infarction. $N$ Engl J Med 2017;376:1234-44.
3 Engstrøm T, Kelbæk H, Helqvist S, et al. Complete revascularisation versus treatment of the culprit lesion only in patients with STsegment elevation myocardial infarction and multivessel disease (DANAMI-3-PRIMULTI): an open-label, randomised controlled trial. Lancet 2015;386:665-71.

4 Masdjedi K, van Zandvoort LJC, Balbi MM, et al. Validation of a three-dimensional quantitative coronary angiography-based software to calculate fractional flow reserve: the fast study. Eurolntervention 2020;16:591-9.

5 Thygesen K, Alpert JS, Jaffe AS, et al. Third universal definition of myocardial infarction. J Am Coll Cardiol 2012;60:1581-98.

6 Spitzer E, McFadden E, Vranckx P, et al. Defining staged procedures for percutaneous coronary intervention trials: a guidance document. JACC Cardiovasc Interv 2018;11:823-32.

7 Garcia-Garcia HM, McFadden EP, Farb A, et al. Standardized end point definitions for coronary intervention trials: the academic research Consortium-2 consensus document. Circulation 2018;137:2635-50.

8 Choi KH, Lee JM, Koo B-K, et al. Prognostic Implication of Functional Incomplete Revascularization and Residual Functional SYNTAX Score in Patients With Coronary Artery Disease. JACC Cardiovasc Interv 2018;11:237-45.

9 Dattilo PB, Prasad A, Honeycutt E, et al. Contemporary patterns of fractional flow reserve and intravascular ultrasound use among patients undergoing percutaneous coronary intervention in the United States: insights from the National Cardiovascular Data Registry. J Am Coll Cardiol 2012;60:2337-9.

10 Parikh RV, Liu G, Plomondon ME, et al. Utilization and outcomes of measuring fractional flow reserve in patients with stable ischemic heart disease. J Am Coll Cardiol 2020;75:409-19.

11 de Waard GA, Hollander MR, Teunissen PFA, et al. Changes in coronary blood flow after acute myocardial infarction: insights from a patient study and an experimental porcine model. JACC CardiovasC Interv 2016;9:602-13.

12 van der Hoeven NW, Janssens GN, de Waard GA, et al. Temporal changes in coronary hyperemic and resting hemodynamic indices in Nonculprit vessels of patients with ST-segment elevation myocardial infarction. JAMA Cardiol 2019;4:736-44.

13 Ciccarelli G, Barbato E, Toth GG, et al. Angiography versus hemodynamics to predict the natural history of coronary stenoses: fractional flow reserve versus angiography in multivessel evaluation 2 substudy. Circulation 2018;137:1475-85.

14 Van Belle E, Rioufol G, Pouillot C, et al. Outcome impact of coronary revascularization strategy reclassification with fractional flow reserve at time of diagnostic angiography: insights from a large French multicenter fractional flow reserve registry. Circulation 2014;129:173-85.

15 Sejr-Hansen M, Westra J, Thim T, et al. Quantitative flow ratio for immediate assessment of nonculprit lesions in patients with ST-segment elevation myocardial infarction-An iSTEMI substudy. Catheter Cardiovasc Interv 2019;94:686-92.

16 Tu S, Westra J, Adjedj J, et al. Fractional flow reserve in clinical practice: from wire-based invasive measurement to image-based computation. Eur Heart J 2020;41:3271-9.

17 Collet C, Onuma Y, Sonck J, et al. Diagnostic performance of angiography-derived fractional flow reserve: a systematic review and Bayesian meta-analysis. Eur Heart J 2018;39:3314-21.

18 Asano T, Katagiri Y, Chang CC, et al. Angiography-Derived fractional flow reserve in the SYNTAX II trial: feasibility, diagnostic performance of quantitative flow ratio, and clinical prognostic value of functional SYNTAX score derived from quantitative flow ratio in patients with 3-Vessel disease. JACC Cardiovasc Interv 2019;12:259-70.

19 Kogame N, Takahashi K, Tomaniak M, et al. Clinical implication of quantitative flow ratio after percutaneous coronary intervention for 3-Vessel disease. JACC Cardiovasc Interv 2019;12:2064-75.

20 Song L, Tu S, Sun Z, et al. Quantitative flow ratio-guided strategy versus angiography-guided strategy for percutaneous coronary intervention: rationale and design of the favor III China trial. Am Heart J 2020;223:72-80. 